\title{
Segmental Elements for the Non-Linear Analysis of Reinforced Concrete Frames
}

\author{
José Leone, Francisco Barrios, Andrés Salas, Juan Martinez \\ Program of Civil Engineering, Universidad del Magdalena \\ Carrera 32 No 22 - 08, Santa Marta, Colombia \\ joseleonerv@miunimagdalena.edu.co; fbarrios@unimagdalena.edu.co
}

\begin{abstract}
Predicting the behavior of reinforced concrete structures subjected to loads exceeding the service range is highly complex mainly due to cracking, material nonlinearity an the composite nature of concrete. In the case of cracking, current design procedures established in the standards suggest the use of a set of factors that arbitrarily reduce the stiffness of the members, trying to give a conservative estimation of the story drift against seismic loads. Regarding service loads, effective inertia approaches have been successfully used for elements in flexure, but application of these methodologies to more complex systems is not straightforward. This study presents the use of segmental elements as an alternative methodology for the non-linear analysis of reinforced concrete frames at a low computational cost, which can be easily implemented within the day-to-day structural design. Results comparison is made with data from experimental tests and with non-linear analysis using finite element software. Load vs. displacement curves obtained show excellent agreement between the segmental element method and the other methodologies.
\end{abstract}

Keywords: Segmental elements, non-linear analysis, reinforced concrete frames, concrete cracking

\section{Introduction}

One of the goals of structural design is to keep the overall response of the structure within a range considered safe for its use. In order to achieve this goal a variety of design procedures have to be implemented to make sure the functioning of the structural members under certain load conditions is safe. When it comes to concrete material tensile and shear cracking are among the principal causes of stiffness degradation, a wrong estimation of these effects can lead to unsafe designs that compromise the stability of the structure or to economic loss as a result of overestimation of the structural members.

There are a number of numerical models that have been previously developed which incorporate the non-linear properties of concrete in the analysis of reinforced concrete structures [1-5]. Some affect the stiffness matrix of the members to match the amount of crack and damage that they exhibit, within an iterative process controlled by equilibrium equations until reaching the ultimate load. Among these methodologies, Dundar and Kara [1] proposed a procedure that agreed with experimental results up to $78 \%$ of the ultimate load. On the other side, more complex models based on finite elements have been developed as well [2], this approach allows a more detailed analysis and evaluation of the local and global structural response, but usually implies a high computational effort making it difficult for its regular use in structural design.

The authors of this study developed a methodology based on segmental elements that takes into account the stiffness variation due to cracking, reinforcement ratio and material nonlinearity along the members. This approach requires less computational effort than more complex procedures, and it has managed to predict displacements and cracking patterns with excellent agreement in comparison with experimental results and finite element models. 


\section{Methodology}

\subsection{Segmental elements}

A segmental member is based on a typical tridimensional frame element with 12 degrees of freedom (DOFs), but formed with multiple segments that might have different cross sectional properties, length and material properties, see Fig. 1 .

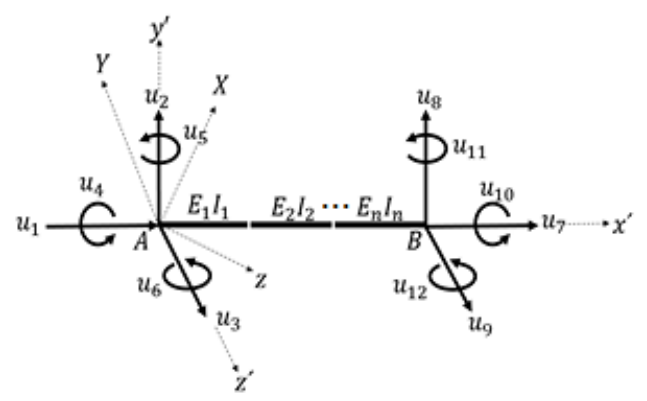

Fig. 1: DOFs of a segmental element with $\mathrm{n}$ segments of different bending stiffness.

After entering the cross sectional properties and length of each segment the sectional response is obtained, and bending moment vs. tangential stiffness matrices (with respect to local axes " $y$ " and " $z$ ") are generated for all the segments within each member present in the structure. These matrices indicate the stiffness of any segment according to the bending moment applied which is also a function of the level of cracking.

\subsection{Local stiffness matrix for a segmental element}

In order to develop the coefficients of axial force of the local stiffness matrix, a unitary axial load is applied to a member formed by $\mathrm{n}$ segments of different cross-sectional area, modulus of elasticity and length. Analogously, the coefficients of torsional force are computed using members formed by $\mathrm{n}$ segments with different shear modulus, torsional constant and length subjected to a unitary torque. On the other hand, integration of the moment function within the element is applied in order to compute the bending and shear positions of the stiffness of a member with $n$ segments of different modulus of elasticity, moment of inertia and length. The moment function is evaluated at each segment within the element to account for the change in the mechanical properties from one segment to the other.

Complete derivation of the stiffness coefficients is by far too extensive to be presented here, therefore only the final expressions of $\mathrm{k} 11$ and $\mathrm{k} 22$ are shown below.

$$
\begin{gathered}
k_{1,1}=\frac{1}{C_{1}} \\
C_{1}=\sum_{i=1}^{n} \frac{l_{i}}{E_{i} A_{i}} \\
k_{2,2}=\frac{C_{2 z}}{C_{z}} \\
C_{2 z}=\sum_{i=1}^{n} \frac{l_{i}}{E_{i} I_{z_{i}}}
\end{gathered}
$$




$$
C_{z}=C_{3 z} C_{5 z}-C_{2 z} C_{6 z}
$$

Where, for the segment $i, \mathrm{~A}_{i}$ is the cross-sectional area, $\mathrm{G}_{i}$ is the shear modulus, $\mathrm{I}_{z i}$ is the second moment of inertia about local axis $\mathrm{z}$, and $l_{i}$ is the length of the segment.

\subsection{Iterative analysis procedure}

The load is applied in small increments to detect changes in the tangential stiffness of any segment within an element in order to modify the global stiffness of the system. Thus, the structure obtained in each cycle might be different from the previous step due to the stiffness reduction in the segmental members. Displacements obtained in each cycle are summed to compute the total displacements of the structure. Conversely, the moments in the segments are cumulative and they are used to detect when the stiffness decrease.

Now, the initial load increment after a stiffness change (trial load) is very small to ensure that the stiffness in any part is not reduced at once when the load is applied, but shows how the new distribution of bending moment in the structure occurs. This initial load step allows to establish a linear relationship between load and bending moment distribution, which is used to identify the first segment within the step (called segment " $R$ ") where the stiffness will change when the magnitude of the loads are increased up to the appropriate level. At this point, displacement and moment values are computed and a new load increment starts. The solution process is illustrated in Fig. 2.

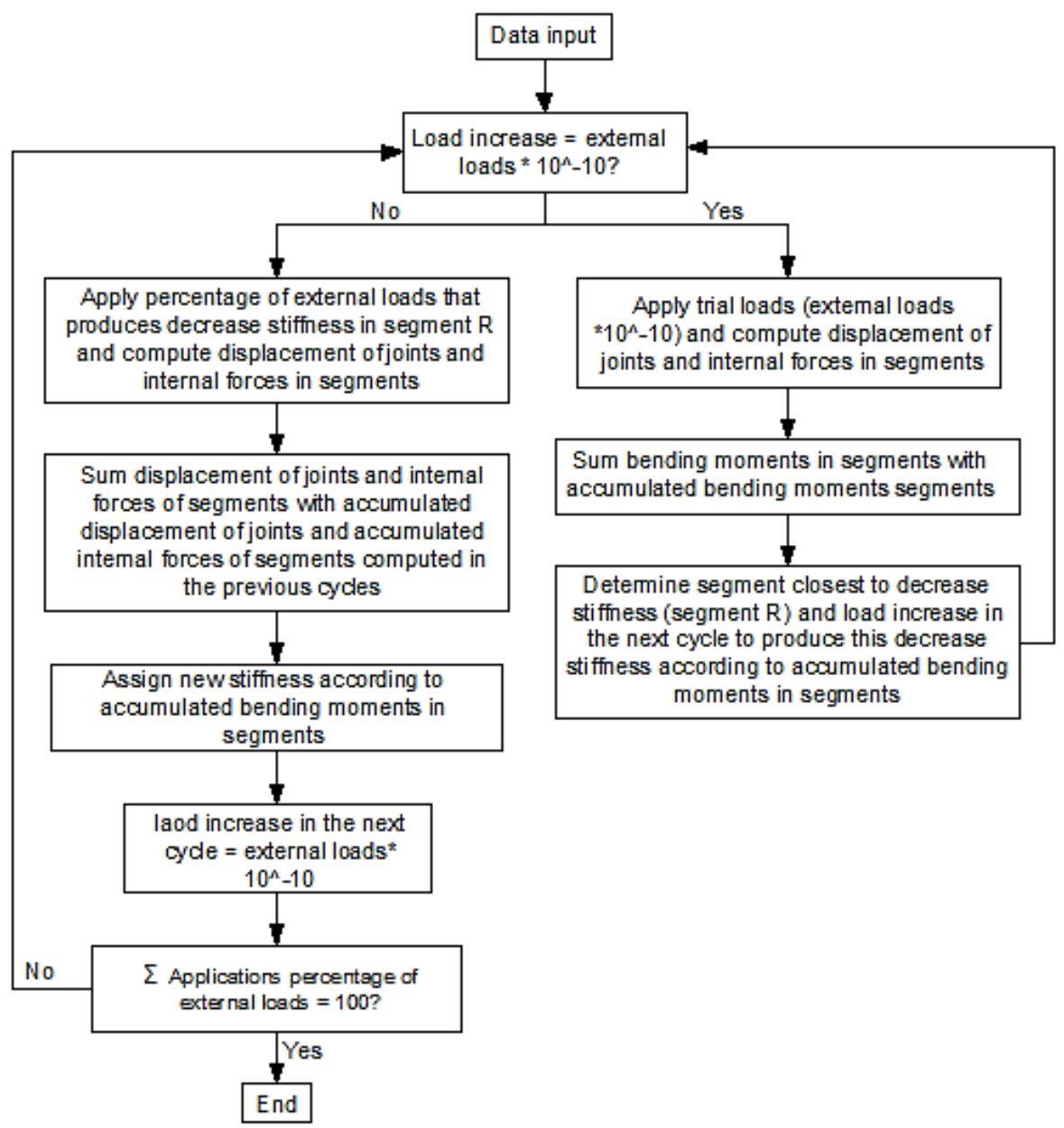

Fig. 2: Scheme of routine of the iterative procedure. 


\section{Results and discussion}

Two different reinforced concrete frames are analysed, one from an experimental test and other with a finite element model, comparison is made based on load vs. displacement curves. Additionally, cracking patterns and cracks distribution are compared with finite element analysis results.

The first analysis performed corresponds to the frame tested by Vecchio and Emara [4], general configuration of the structure is shown in Fig. 3, and analysis results are compared with prior studies that employ alternative approaches [1], see Fig. 4.

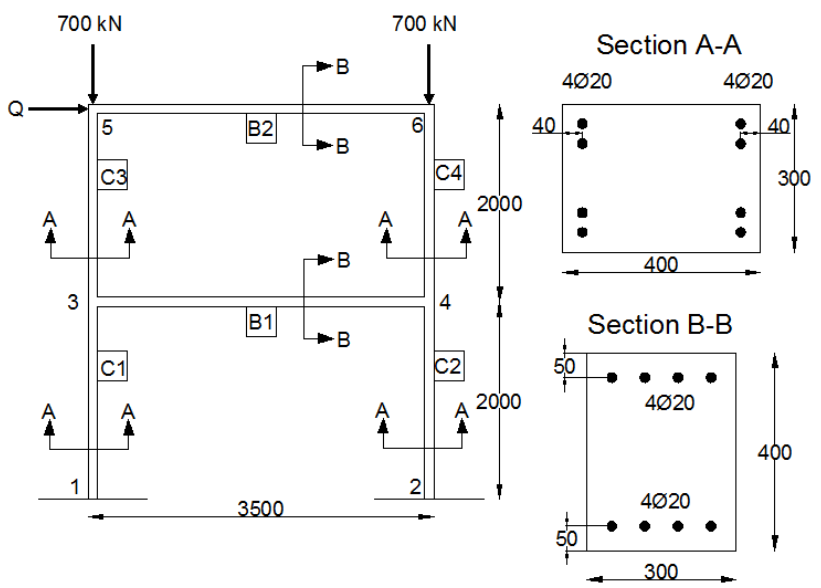

Fig. 3: Reinforced concrete frame tested by Vecchio and Emara [4] (dimensions in mm).

Although the procedure proposed by Vecchio and Emara [4] predicts the displacement at the ultimate load with great accuracy, their load vs. displacement curve follows a path higher than the experimental response. On the other hand, results obtained by Dundar and Kara [1] match the real behavior adequately up to about $78 \%$ of the ultimate load, from that point there is a significant difference between the theoretical and real response. Conversely, the segmental element methodology closely represents the experimental results up to $98 \%$ of the ultimate load.

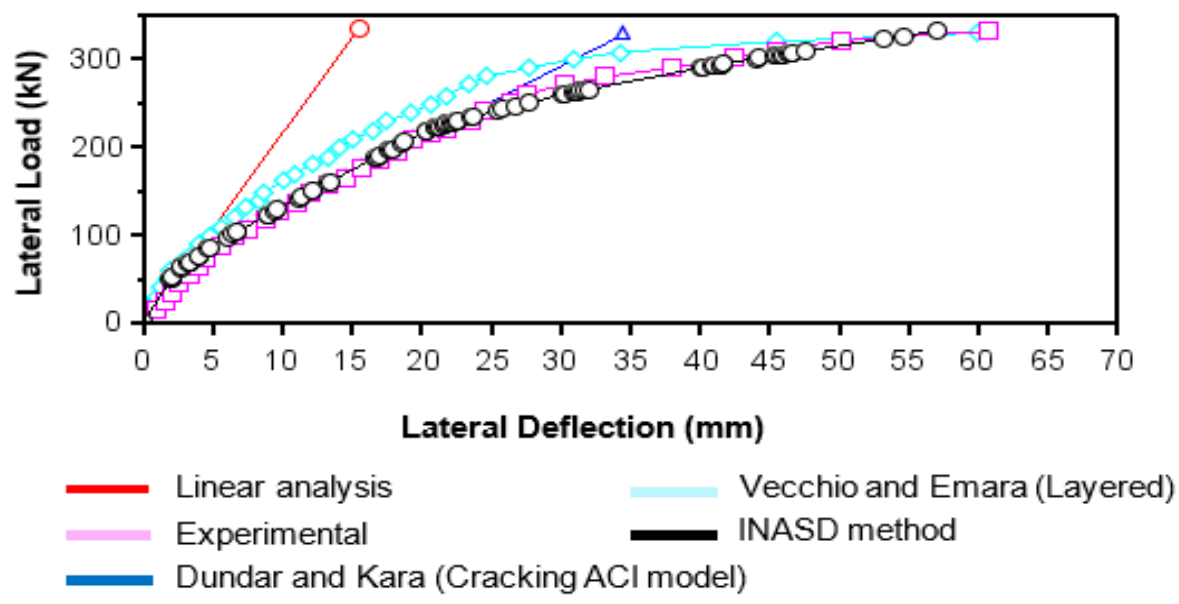

Fig. 4: Results for lateral deflection of Node 5.

For the second case, a one-story and two-bays reinforced concrete frame with different column lengths (Figs. 5 and 6) is analyzed using the Concrete Damaged Plasticity Model (CDPM) incorporated in Abaqus Inc. finite element analysis software[6]. 


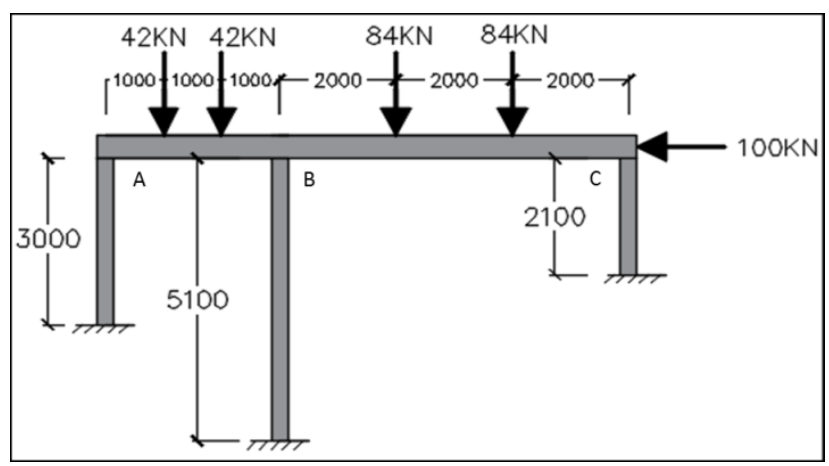

Fig. 5: Loads applied and general dimensions (mm).

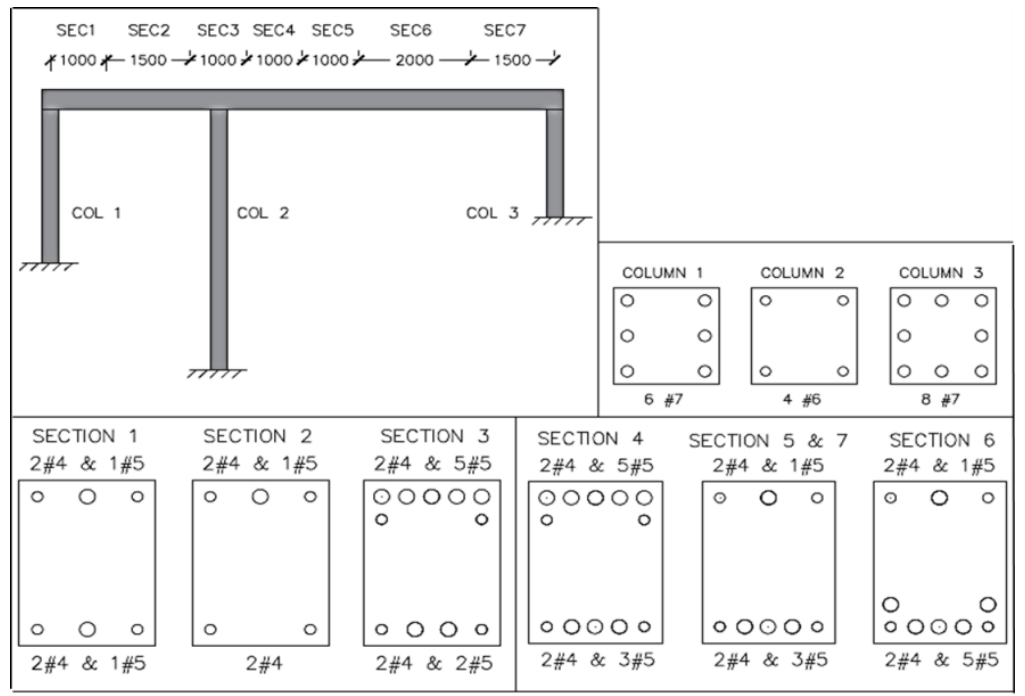

Fig. 6: Cross-sections and reinforcement steel.

Material constitutive model of concrete is defined based on prior studies [7, 8] and from the CEB [9] standards, the fracture energy criterion proposed by Hillerborg et al. [10] accounts for the post-failure behavior of the material. Values suggested by the Abaqus Inc. Analysis user's manual [6] are used for the definition of the failure surface and the behavior of steel is assumed elastic-perfectly plastic with $\mathrm{E}=200 \mathrm{GPa}$ and $\mathrm{f}_{\mathrm{y}}=420 \mathrm{MPa}$. Tables 1 and 2 show a summary of these parameters.

Table 1: Parameters for the definition of the constitutive behavior of concrete material.

\begin{tabular}{|c|c|c|c|c|}
\hline $\mathbf{f}^{\prime} \mathbf{c}(\mathrm{MPa})$ & $\boldsymbol{d}_{\max }(\mathbf{m m})$ & $\mathbf{G}_{\mathbf{F}}$ & $\mathbf{f}_{\mathbf{t}}(\mathrm{MPa})$ & $\mathbf{E}(\mathrm{MPa})$ \\
\hline $\mathbf{2 8}$ & 32 & 0.080 & 2.70 & 25000 \\
\hline
\end{tabular}

To avoid convergence problems a visco-regularization factor $(\mu)$ of 0.0001 is included, maximum mesh size chosen is $50 \mathrm{~mm}$ from a mesh sensitivity analysis. The concrete material is simulated by $3 \mathrm{D}$ first order finite solid elements and consists in 8 node blocks with lineal interpolation in each direction and reduced integration (C3D8R). The steel elements are Truss 3D type with two nodes (T3D2) and the reinforcement bars are embedded in the concrete material.

Table 2: Parameters used for the definition of the failure surface.

\begin{tabular}{|c|c|c|c|c|}
\hline$\psi$ & $\mathrm{K}_{\mathrm{c}}$ & $\sigma_{\mathrm{b} 0} / \sigma_{\mathrm{c} 0}$ & $\epsilon$ & $\mu$ \\
\hline 13 & $2 / 3$ & 1.16 & 0.1 & 0.0001 \\
\hline
\end{tabular}


Results are compared in terms of load percentage vs. displacement curves as shown in Fig. 7. General behavior of the structure is linear-elastic in both models until approximately $30 \%$ of the load; from there the slope is reduced due to the effects of the accumulation of cracks and the corresponding loss of stiffness at the base of column 3 . The slope in the finite element analysis gradually changes while there is a sharper change using the segmental elements with variable stiffness coefficients method. Despite the difference in how the onset of cracking affects the shape of each of the curves, the responses stayed close throughout the loading. Final displacement predicted by the segmental element method shows excellent agreement with the one obtained using finite element analysis, which required a higher computational cost.

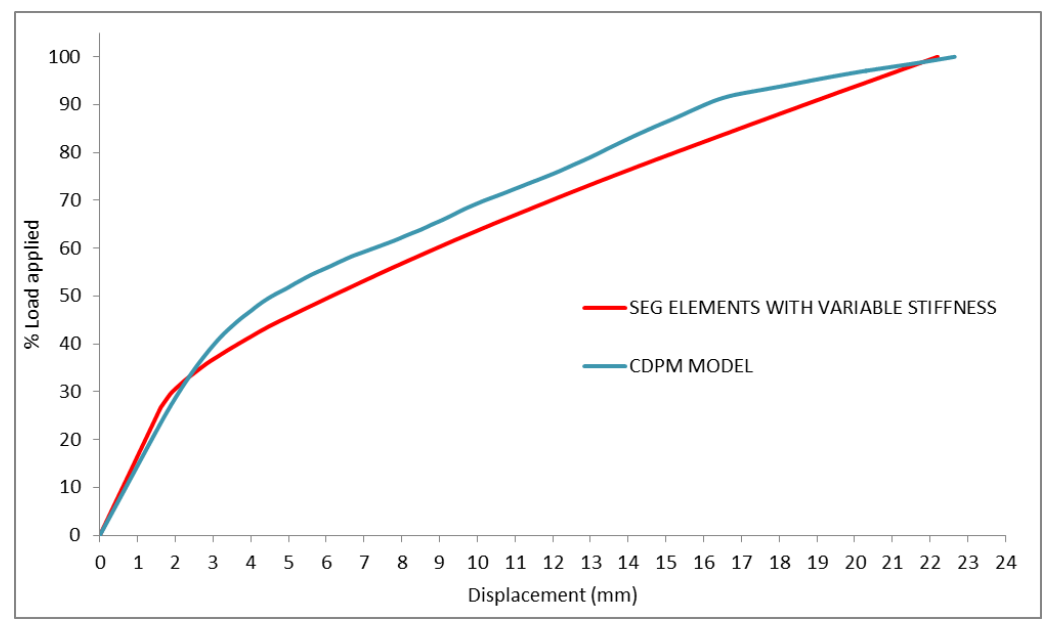

Fig. 7: Results for lateral displacement of Node A.

Furthermore, the location and amount of damage at the first crack and at the ultimate load predicted by the segmental elements method is very similar in both procedures, see Fig 8.

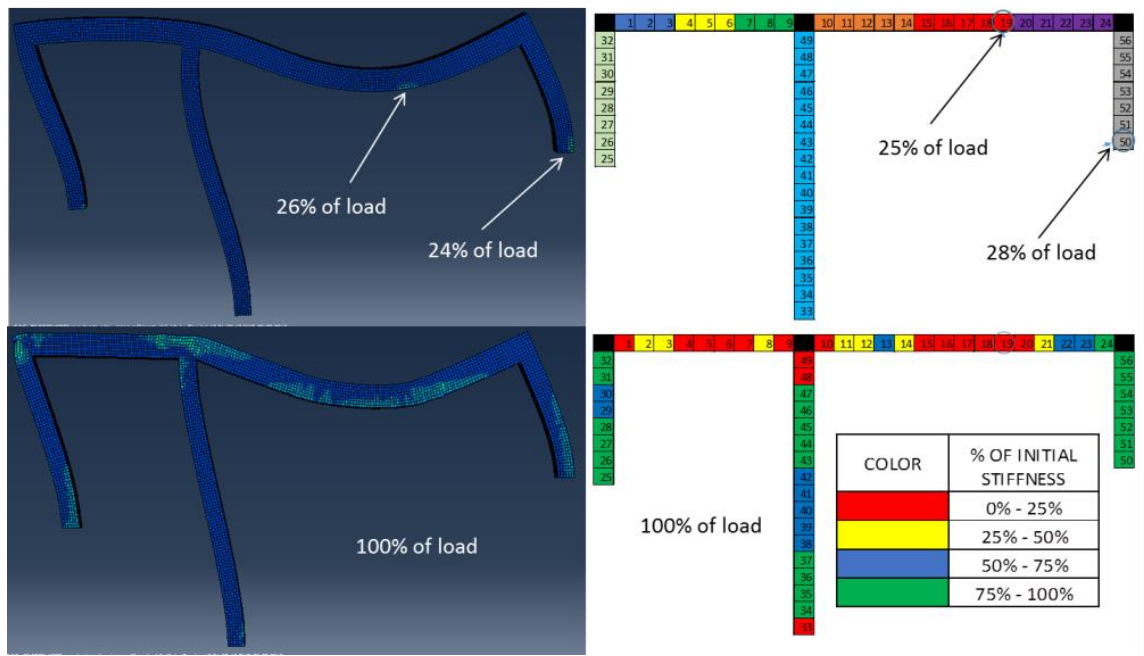

Fig. 8: First plastic deformations (cracking) detected and plastic deformations at end of the test. Abaqus (left) - Segmental elements (right). 


\section{Conclusions and future research}

The segmental element procedure was able to describe with excellent agreement the load versus displacement response from the experimental test up to load levels over $90 \%$ of the ultimate, at a relatively low computational cost since the implementation of segmental elements does not increase the size of the stiffness matrix to account for the cracking within the members. Additionally, a convergence study showed that the inclusion of a larger number of segments did not alter the numerical results meaningfully.

The location and percentage at the onset of cracking are remarkably close and the final displacement differed in less than $5 \%$ between both methodologies. Additionally, the cracking pattern in the finite element model is very similar to the stiffness distribution in the segmental elements at ultimate.

Stress concentration at the supports, cracking at the nodes and the concrete constitutive model may be the cause of some the differences found in the non-linear response of the structure between both methods.

In the present study, the sectional analysis to account for the stiffness degradation within the members was limited to uniaxial bending. However, members in biaxial flexure and compression (such as columns in a three-dimensional structure) experience a more complex behaviour where cracking in one direction affects the stiffness and cracking on the transversal direction and the torsional response as well. Nevertheless, the segmental elements and the iterative solution presented are independent of the procedure selected for obtaining the sectional response of the segments which can be replaced with ease in the algorithm.

\section{Acknowledgements}

The authors wish to express their most sincere gratitude towards the structural engineer Jaime de León whose work initiated this research. Jaime's collaboration and expertise was critical during the mathematical formulation, and without it, the realization of this study would not have been possible. The authors also wish to thank the Universidad Del Magdalena for its support throughout this project.

\section{References}

[1] C. Dundar and I.F. Kara "Three-dimensional analysis of reinforced concrete frames with cracked beam and column elements," Engineering Structures, 2007.

[2] RSH. Al-Mahaidi "Nonlinear finite element analysis of reinforced concrete deep members," Department of Struct. Engrg. Cornell University, Report No: 79-1, pp. 357, 1978.

[3] M.E. Marante and J. Flórez-López, "Three-dimensional analysis of reinforced concrete frames based on lumped damage mechanics," International Journal of Solids and Structures, vol. 40, no. 19, pp. 5109-5123, 2003.

[4] F. Vecchio and M. B. Emara, "Shear deformations in reinforced concrete frames," Structural Journal, vol. 89, no. 1, pp .46-56, 1993.

[5] C. M. Chan, N. C. Mickleborough, F. Ning, "Analysis of cracking effects on tall reinforced concrete buildings," Journal of Structural Engineering, ASCE, 2000.

[6] ABAQUS, Inc. v. 6.12-1. Analysis user's manual, 2012.

[7] F. López-Almanza, B. Alfarah, S. Oller, "Numerical simulation of RC frame testing with damage plasticity model. Comparison with simplified models," Second European Conference On Earthquake Engineering And Seismology, Istanbul, Aug. 25-29, 2014.

[8] Y. Sümer, and M. Aktaş, "Defining parameters for concrete damage plasticity model," Challenge Journal of Structural Mechanics, vol. 1, no. 3, pp. 149-155, 2015.

[9] Comite Euro-International du Beton. Manual on cracking and deformation. Bull Inform, 158-E, 1985.

[10] A. Hillerborg, M. Modéer, and P.E. Petersson, "Analysis of crack formation and crack growth in concrete by means of fracture mechanics and finite elements," Cement and concrete research, vol. 6, no. 6, pp. 773-781, 1976. 